Research Paper

\title{
CEP2 Attenuates Myoblast Differentiation But Does Not Affect Proliferation
}

\author{
Ming Wu ${ }^{1}$, Gongshe Yang ${ }^{2}$, Yaosheng Chen ${ }^{1}$, Xingyu Zhou ${ }^{1}$, Hu Chen ${ }^{1}$, Mingsen $\mathrm{Li}^{1}$, Kaifan Yu ${ }^{1}$, Xumeng \\ Zhang ${ }^{1}$, Shuihua Xie ${ }^{1}$, Ying Zhang1, Guiyan $\mathrm{Chu}^{2}$, Delin $\mathrm{Mo}^{1} \bowtie$ \\ 1. State Key Laboratory of Biocontrol, School of Life Sciences, Sun Yat-Sen University, Guangzhou 510006, China; \\ 2. Laboratory of Animal Fat Deposition and Muscle Development, College of Animal Science and Technology, Northwest A\&F University, \\ Yangling, China.
}

\begin{abstract}
$\square$ Corresponding author: Delin Mo. Address: State Key Laboratory of Biocontrol, School of Life Sciences, Sun Yat-Sen University, Guangzhou 510006, China. Email: modelin@mail.sysu.edu.cn; Tel: (86) 20-39332991; Fax: (86) 20-39332940.

(C) Ivyspring International Publisher. This is an open-access article distributed under the terms of the Creative Commons License (http://creativecommons.org/ licenses/by-nc-nd/3.0/). Reproduction is permitted for personal, noncommercial use, provided that the article is in whole, unmodified, and properly cited.
\end{abstract}

Received: 2014.01.01; Accepted: 2014.03.11; Published: 2015.01.01

\begin{abstract}
CEP2 (CDC42EP2) is a member of the CDC42 subfamily that belongs to the Rho family. The Rho family plays an important role in a variety of cellular processes including skeletal myogenesis. Here, we find the expression of CEP2 increased significantly during $\mathrm{C} 2 \mathrm{C} 12$ myogenesis. Overexpression of CEP2 could attenuate myoblast differentiation, while knockdown of CEP2 by siRNA results in enhancing myogenesis. Furthermore, we demonstrate for the first time that CEP2 attenuates myoblast differentiation via suppression of muscle regulatory factors (MRFs) rather than influencing myoblast proliferation. These results indicate that CEP2 acts as a repressor during myogenesis, which provides new insights into the role of CEP2 in muscle development.
\end{abstract}

Key words: CEP2; myogenesis; skeletal muscle; myoblast proliferation.

\section{Introduction}

The formation of mature skeletal muscle is a multistep process wherein pluripotent mesodermal cells commit to myoblasts, and then myoblasts withdraw from the cell cycle and fuse into plurinucleated myotubes [1,2]. The development of skeletal muscle is mainly orchestrated by a family of muscle-regulatory factors (MRFs) including myoD, myf5, myogenin, and mrf4 $[3,4]$. At the onset of myoblast differentiation, proliferating myoblasts express myf5 and $m y o D$. When myoblasts are cultured in differentiation medium, myoD induce termination of myoblast proliferation and the expression of myogenin. Besides, the process that coordinates myoblast proliferation and myoblast differentiation needs down-regulation of cell cycle activators such as cyclins and cdks and up-regulation of cell cycle inhibitors such as $\mathrm{Rb}, \mathrm{p} 21$, p27 and p57 [5-9]. Indeed, a crosstalk between myoblast proliferation and differentiation is important during myogenesis.
MRFs are targets of various signaling pathways including the Rho family of small GTPases which consists of three subfamilies (Rho, Rac, and Cdc42) [10-16]. During myogenesis, Cdc42 (cell division cycle 42 homolog) regulates myoblast fusion by recruiting F-actin and other molecules to the sites of cell-cell contact in vitro and in vivo $[17,18]$. To be competent for fusion, myoblasts extend lamellipodium and filopodia for contacting with neighbors [19], which is necessary to recognize each other. Interestingly, CEP2 involved in the formation of pseudopodia by interacting with Cdc42. In addition, co-expression of CEP2 with dominant negative mutant CDC42 or expressing a Cdc42/Rac interactive binding domain mutant of CEP2 didn't induce long pseudopodia formation in fibroblasts [20]. These results suggest CEP2 has a function in myoblast shape changes, recognition and fusion. Previous study has identified that CEP2 is down-regulated in tongue muscle from myogenin ${ }^{-/-}$ 
embryos using microarray analysis [21], which implies that CEP2 plays a role in skeletal muscle differentiation. Another study reveals CEP2 mRNA is decreased in miR-29a mutant mouse progenitors using gene array, indicating CEP2 links to miR-29a (a promoter) in myogenesis [22, 23]. Transcriptional profiling shows that CEP2 may play pivotal roles in cytokinesis of human cardiomyocytes [24], which shares some of the common myogenic factors. Besides, CEP2 is also known as a Rho GTPase [20, 25-26], which can be regarded as a molecular switch controlling cell behavior [12]. Therefore, the role of CEP2 in myogenesis attracts our interest.

Although the role of CEP2 in fibroblasts is well elaborated, its biological function in myoblasts remains unknown. The aim of the present study was to characterize the CEP2 in myogenesis, and gain insight into its potential biological role in muscle development by overexpressing and knocking down it in C2C12 myoblast.

\section{Materials and Methods}

\section{Plasmids and Generation of Lentivirus Cell Lines}

The cDNA of mouse CEP2 were separately cloned into $p c D N A 3.1$ vector and lentivirus packing vector pl411G GLUC (Invitrogen, Shanghai, China). The other lentiviral components including $p M D 2 G$ and PSPAX2 were extracted using ultrapure endotoxin-free extraction kits (Omega, Guangzhou, China).

The lentivirus vectors were prepared by transient transfection of 293T cells using the calcium phosphate precipitation method. After incubation for 48 , 60 and $72 \mathrm{~h}$, the cell supernatant containing virus-like particles was collected. Subsequently, the viral titers were determined after condensation. When $\mathrm{C} 2 \mathrm{C} 12$ cell confluence reached $50-60 \%$ in 6-well plates, C2C12 cells were infected with lentivirus-based CEP2 and an empty lentivirus vector (CON) for 12 hours. One or two days later, the GFP expression level was measured by fluorescence microscopy as the transfection efficiency. Cells were then harvested and screened with flow cytometer (Becton Dickinson, Franklin Lakes, NJ, USA). The positive cells obtained were passed, harvested and screened for 6 times. Finally the differentiated cells were used for mRNA and protein level analysis. All transfections were performed in triplicate for each experiment.

\section{Cells Culture and Differentiation Induction}

Mouse $\mathrm{C} 2 \mathrm{C} 12$ myoblasts were cultured in high-glucose DMEM (Gibco, Guangzhou, China) with $10 \%(\mathrm{v} / \mathrm{v})$ fetal bovine serum (GM) and were main- tained at $37{ }^{\circ} \mathrm{C}$ in $5 \% \mathrm{CO}_{2}$ incubator. To induce differentiation, culture medium was changed to DMEM with $2 \%$ horse serum (DM) (Gibco) when cells reached confluence. The culture medium was replaced at 2-day intervals before the end of the checkpoint.

\section{Cell Transfection}

The $\mathrm{C} 2 \mathrm{C} 12$ cells were seeded in twelve-well or six-well plates. After 12 hours, when cell confluence reached $50 \% \sim 60 \%$, the plasmids or siRNA pools were transfected into C2C12 cells using Lipofectamine 2000 transfection reagents (Life Technologies, Shanghai, China) or DharmaFECT siRNA transfection reagents (Thermo Fisher, Guangzhou, China) following the manufacturer's instructions. Nucleic acids and transfection reagents were diluted by Opti-MEM I without Serum Medium (Gibco). The sequences of siRNA pools were listed in Supplementary Material: Table S1.

\section{Real Time RT-PCR (qPCR)}

Total RNA was extracted from cells with TRIzol reagent (Life Technologies) and treated with DNase I (Promega, Beijing, China). The concentration and quality of RNA were assessed by NanoDrop ND-1000 (Thermo, Waltham, MA, USA) and denatured gel electrophoresis. Reverse transcription was performed using AMV reverse transcriptase (Promega). The qPCR reaction was carried out in the LightCycler 480 II system (Roche, Basel, Switzerland). The sequences of qPCR primers can be found in Supplementary Material: Table S2. Statistical analysis of mRNA relative expression was performed with Student's $t$ test. The analytic method of $2^{-\Delta \Delta C t}$ was used, $\Delta \Delta C t=$ [target gene (treatment group) / target gene (control group)] / [house-keeping gene (treatment group) / house-keeping gene (control group)]. ${ }^{*} p<0.05$; ${ }^{* *} p<0.01 ; \mathrm{n} \geq 3$ ( $\mathrm{n}$ : Biological replicates).

\section{Western blot}

C2C12 cells were treated with Lysis Buffer (Beyotime Biotechnol, Shanghai, China), which was premixed with PMSF (100:1). The proteins at supernatant were separated by SDS-PAGE, followed by protein transfer to polyvinylidene fluoride membranes using Mini Trans-Blot Cell (Bio-Rad, Hercules, CA, USA). The proteins were detected by primary antibodies specific for Myf5 (\#sc-302, Santa Cruz, CA, USA), MyoD (\#sc-304, Santa Cruz), MRF4 (\#sc-301, Santa Cruz). $\beta$-actin (\#4970s, CST, Massachusetts, USA ) served as loading controls in all western blot assays. The anti-rabbit secondary antibody (\#7074s, CST) was used in this assay. Blots were visualized using a commercial enhanced chemiluminescene (ECL) detection Kit (Thermo Scientific, Beijing, Chi- 
na). All results of western blot were calculated and presented as mean \pm S.E.M. ${ }^{*} p<0.05 ;{ }^{* *} p<0.01 ; \mathrm{n}=3$.

\section{Immunofluorescence}

After transfection and differentiation, $\mathrm{C} 2 \mathrm{C} 12$ cells were harvested and detected by immunofluorescence following the manufacturer's instructions. Primary anti-myosin (skeletal, fast) antibody (\#ab7784, Abcam, Hong Kong, China), anti-CEP2 antibody (\#ab173012, Abcam), anti-myogenin antibody (\#IMG131, Novus, Littleton, CO) and secondary antibody labeled with Alexa Fluor 555 fluorochrome (red) (Invitrogen) or labeled with FITC 488 fluorochrome (green) (Invitrogen) were used. Images were captured with a fluorescence inverse microscope (ZEISS, Heidenheim, Germany) (5 random fields were captured for each treatment group). Confocal microscopy (Leica Microsystems, Deerfield, IL) was used in the subcellular location of CEP2 protein experiment. $\mathrm{n}=3$.

\section{Cell Proliferation Analysis}

Analysis of Cell Cycle by Flow Cytometry: After transfection for 24 hours, suspension cells were fixed in ice-cold $70 \%(\mathrm{v} / \mathrm{v})$ ethanol overnight. 50 $\mathrm{mg} / \mathrm{ml}$ propidium iodide solution $(\mathrm{PI})$ (which contains $100 \mathrm{mg} / \mathrm{ml}$ RNase A and $0.2 \%$ (v/v) Triton X-100) was added to suspension cells, then those cells

A
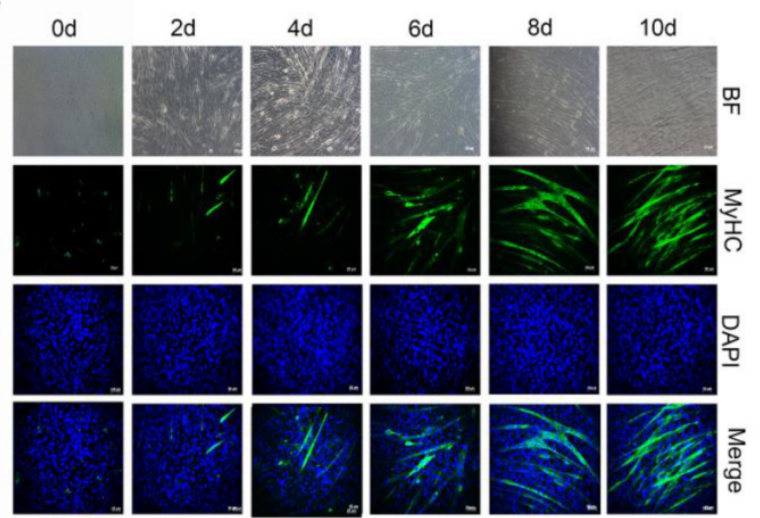

B
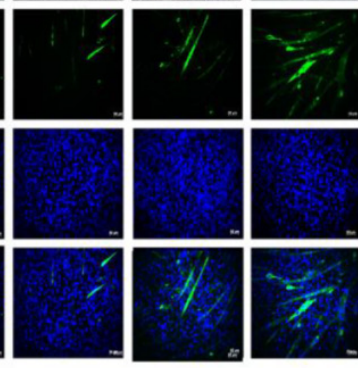

D
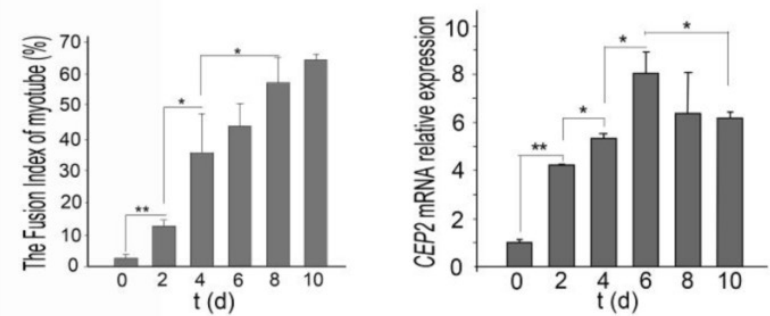

were incubated at $4{ }^{\circ} \mathrm{C}$ for $30 \mathrm{~min}$. Proliferative index was analyzed by BD FACSCalibur (Becton Dickinson, Franklin Lakes, NJ, USA) and ModFit software (Verity Software House, Topsham, ME, USA). The percentage of mitotic cells from a total of 10000 cells examined (n $=3$ ) stands for the proliferative index.

EdU assay: After cells were transfected for 24 hours, EdU reagents (RIBOBIO, Guangzhou, China) (final concentration, $10 \mathrm{mM}$ ) were added for 4 hours. $4 \%$ paraformaldehyde was used for cell fixation, the proportion of EdU-positive cells were detected by fluorescence inverse microscope ( 5 random fields were captured for each treatment group, $n=3$ ).

AlamlaBlue assay: 6 hours post-transfection, alamlaBlue reagents (Invitrogen) (final concentration, $10 \% \mathrm{v} / \mathrm{v}$ ) were added into fresh complete medium (n $=6$ ). The fluorescence value was detected by a microplate reader (BioTek, Vermont, USA) at 6 or 12-hour intervals.

\section{Statistical analysis}

All results are presented as mean \pm standard errors of the means (SEM) based on at least three independent experiments. Unless additional notion, the differences between groups were analyzed with a Student's two-tailed t test when only two groups were compared or ANOVA when more than two groups

C

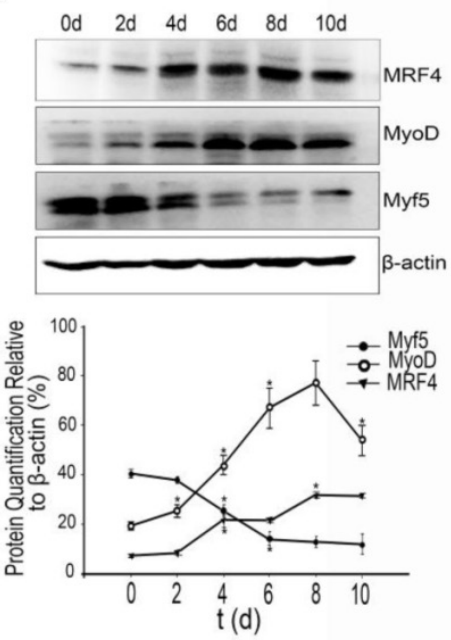
were compared. ${ }^{*} p<0.05$; ${ }^{* *} p<0.01 ; \mathrm{n} \geq 3$.

\section{Results}

\section{CEP2 is increased during $22 \mathrm{C} 12$ my-} oblast differentiation

The first issue that we sought to address was whether CEP2 participates in $\mathrm{C} 2 \mathrm{C} 12$ myoblast differentiation. For this purpose, we established a $\mathrm{C} 2 \mathrm{C} 12$ induction model that was confirmed by immunofluorescence of MyHC (a muscle-specific protein) (Figure 1A). The fusion index of myotube increased regularly (Figure 1B). During differentiation, Myf5 protein exerted high expression at the initiation of $\mathrm{C} 2 \mathrm{C} 12$ differentiation, and declined rapidly after 2

Figure 1. CEP2 was upregulated during $\mathrm{C} 2 \mathrm{C} 12$ myoblast differentiation. (A) A normal model of $\mathrm{C} 2 \mathrm{Cl} 2$ cells differentiation was established using DMEM with $2 \%$ horse serum (DM). Every two days, MyHC protein was detected by anti-myosin (skeletal, fast) antibody (green). The nuclei were stained with DAPI (blue). BF: bright field. Scale bar $=50 \mu \mathrm{m}$. (B) The fusion index (percentage of the number of nuclei residing in the MyHC-positive cells divided by the total number of the nuclei) was calculated during differentiation. ${ }^{*} p<0.05 ; * *<<0.01, n=3$. (C) Western blotting analysis of myogenic factors during differentiation. $* p<0.05 ; n=3$. (D) CEP2 expression in $\mathrm{C} 2 \mathrm{Cl} 2$ cells during differentiation. The level of mRNA was in relation to that on 0 days. $* p<0.05 ; * *<<0.01, \mathrm{n}=3$ (three independent replicates per group). 
days. Unlike Myf5, MyoD protein gradually was up-regulated, but declined after 8 days. MRF4 protein exhibited continuous increase (Figure 1C). CEP2 expression was continuous increased significantly, and then gradually decreased at the end of $\mathrm{C} 2 \mathrm{C} 12$ differentiation (Figure 1D).

\section{Up-regulation of CEP2 attenuates myoblast differentiation}

To address whether CEP2 gene influences myoblast differentiation, we detected muscle-specific gene expression after transfection with pcDNA3.1-CEP2. CEP2 was up-regulated significantly in $\mathrm{C} 2 \mathrm{C} 12$ cells (Figure 2A). As a result, the mRNA level of myf5, myoD, myogenin, mrf4, troponin $\mathrm{T} 2$ and $\mathrm{MyHC} 2 b$ were decreased significantly (Figure 2A). Myf5, MyoD, MRF4 expression were decreased as well (Figure 2B). When the cells cultured in DM for 4
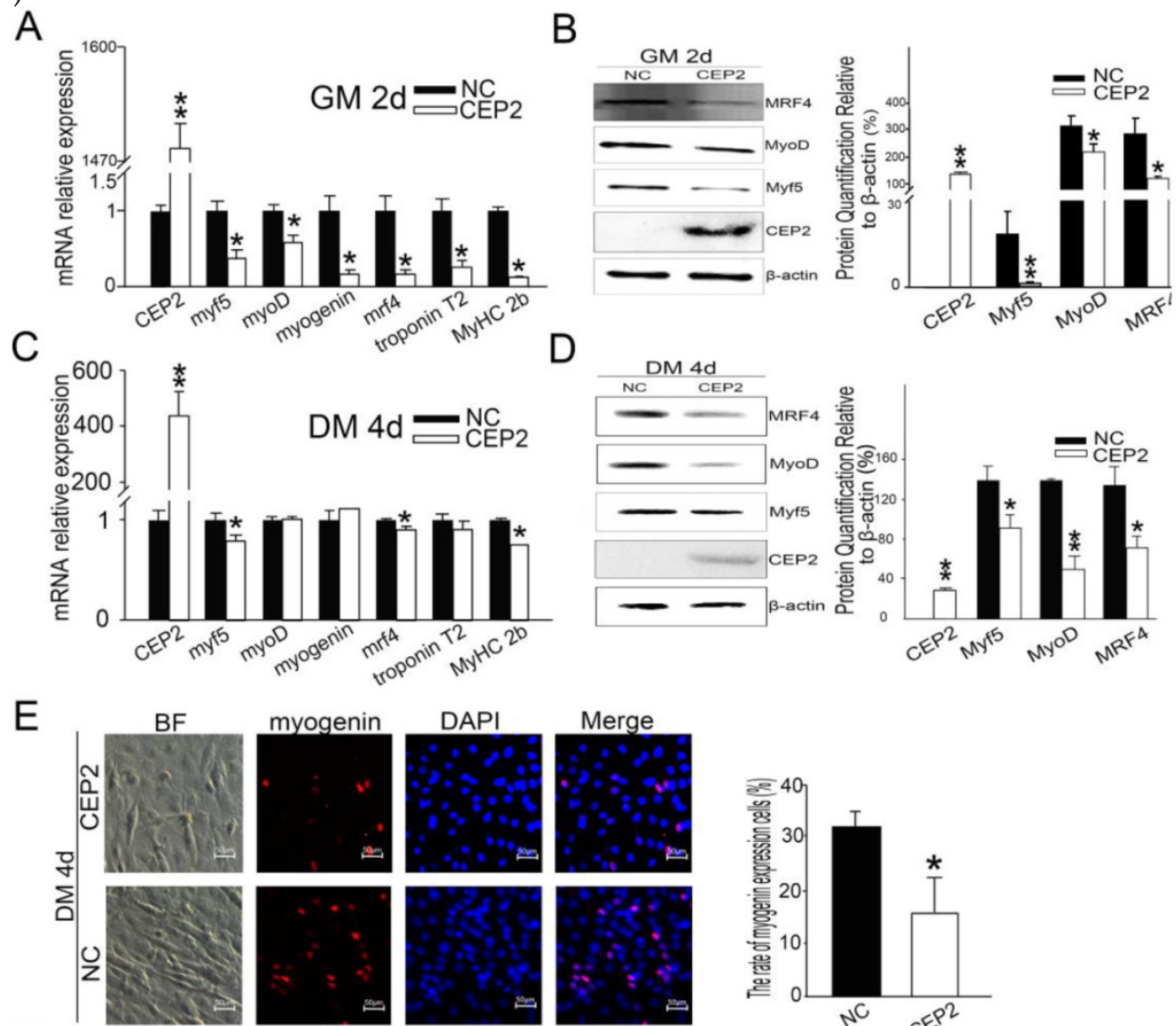

F

$\mathrm{BF}$
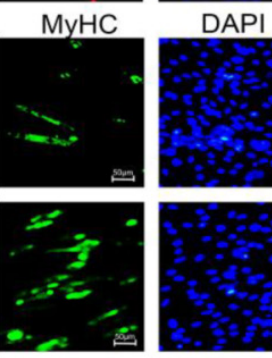

Merge
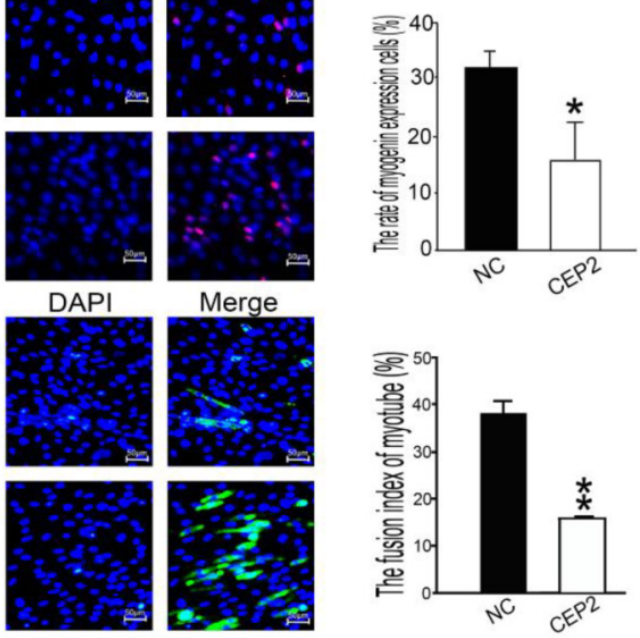

Figure 2. Overexpression of CEP2 mediated by plasmids inhibited myoblast differentiation. RNA (A) and protein (B) of CEP2 and myogenic factors were analyzed in GM after 2 days post-transfection. Results were presented as mean \pm S.E.M. After differentiation for 4 days, the mRNA (C) and protein (D) of myogenic factors were analyzed again. Myogenin (red) (E) and MyHC (green) (F) were detected using immunofluorescence in DM. The rate of myogenin expression cells was calculated. Scale bar = 50 $\mu \mathrm{m}$. NC: native control (pcDNA3.I); CEP2 (pcDNA3.I-CEP2). $*_{p}<0.05$; ** $p<0.01 ; \mathrm{n} \geq 3$. 
Compared with the plasmid, lentivirus-mediated transfection presented more stable performance and was therefore employed to study the inhibitory effect of CEP2 on differentiation. Consequently, CEP2 showed significant up-regulation both in GM on the $2^{\text {nd }} \mathrm{d}$ and in DM on the $10^{\text {th }} \mathrm{d}$ (Supplementary Material: Figure S2 A and B). These results presented more stable expression of CEP2. In GM on the $2^{\text {nd }} \mathrm{d}$, the mRNA level of myf5, myoD, myogenin and $M y H C 2 b$ were decreased significantly (Figure 3A). Myf5, MyoD and MRF4 were down-regulated significantly (Figure 3B). After differentiation for 4 days, the mRNA level of myoD, myogenin, mrf4 and MyHC $2 b$ were decreased significantly (Figure 3C). Myf5, MyoD and MRF4 were all reduced (Figure 3D). The rate of myogenin expression cells decreased, demonstrating that the protein level of myogenin de- clined (Figure 3E). To confirm the inhibitory role of $C E P 2$, MyHC proteins were detected on the $4^{\text {th }}, 6^{\text {th }}, 8^{\text {th }}$ and $10^{\text {th }}$ day of post-transfection. As a result, the expression of MyHC declined at four stages mentioned above, demonstrating that myoblast differentiation was attenuated (Figure 4). Furthermore, this kind of inhibition mainly occurred in earlier period of differentiation (Figure 4A). After differentiation for 10 days, MyoD protein increased significantly (Supplementary Material: Figure S2 E and F), although the expression of myf5, mrf4 and MyHC $2 b$ decreased significantly (Supplementary Material: Figure S2 D). It suggested that the expression of CEP2 can result in differentiation delay of myoblasts after 10 days but does not inhibit myoblast differentiation completely. In conclusion, up-regulation of CEP2 could attenuate myoblast differentiation.
A

C
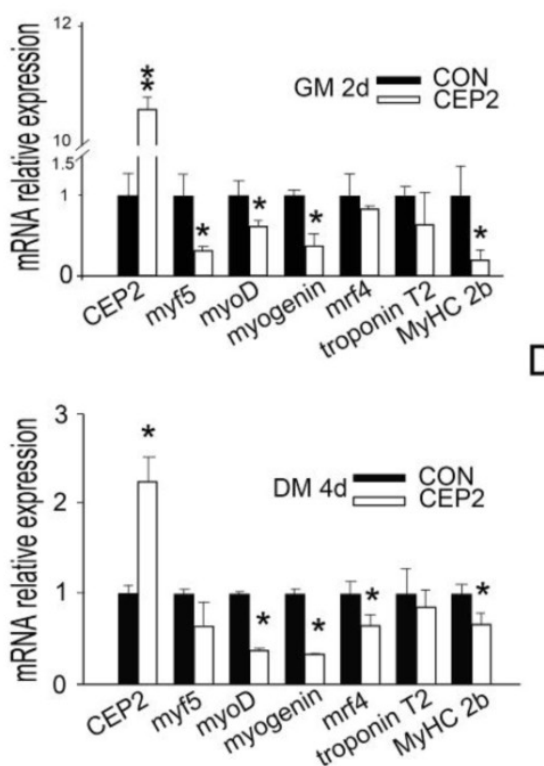

E
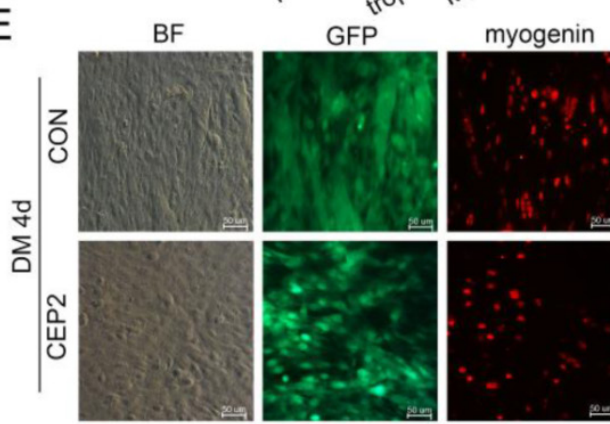

B

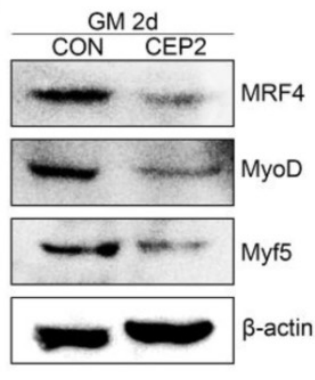

D

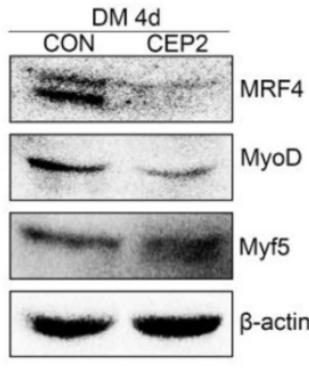

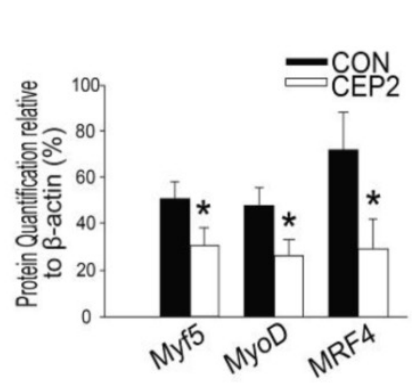

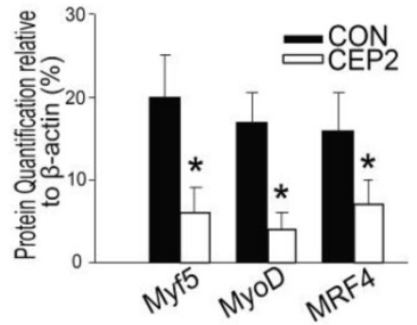

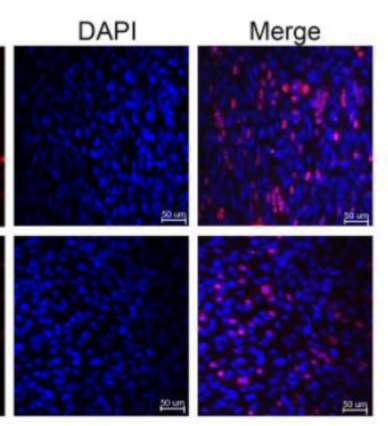

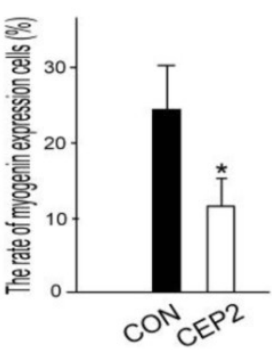

Figure 3. Up-regulation of CEP2 mediated by lentivirus inhibited myoblast differentiation. $\mathrm{QPCR}(\mathrm{A})$ and (B) western blotting for myogenic factors of CEP2-expressing cells and control cells were detected in GM on the $2^{\text {nd }} \mathrm{d}$. After differentiation for 4 days, the mRNA (C) and protein (D) of myogenic factors were analyzed again. (E) The rate of myogenin expression cells was analyzed with immunofluorescence. Scale bar $=50 \mu \mathrm{m}$. CON: native control (an empty lentivirus vector); CEP2 (lentivirus-based CEP2). GFP: represents lentivirus infection efficiency. ${ }^{*} p<0.05 ; * * p<0.01 ; n \geq 3$. 


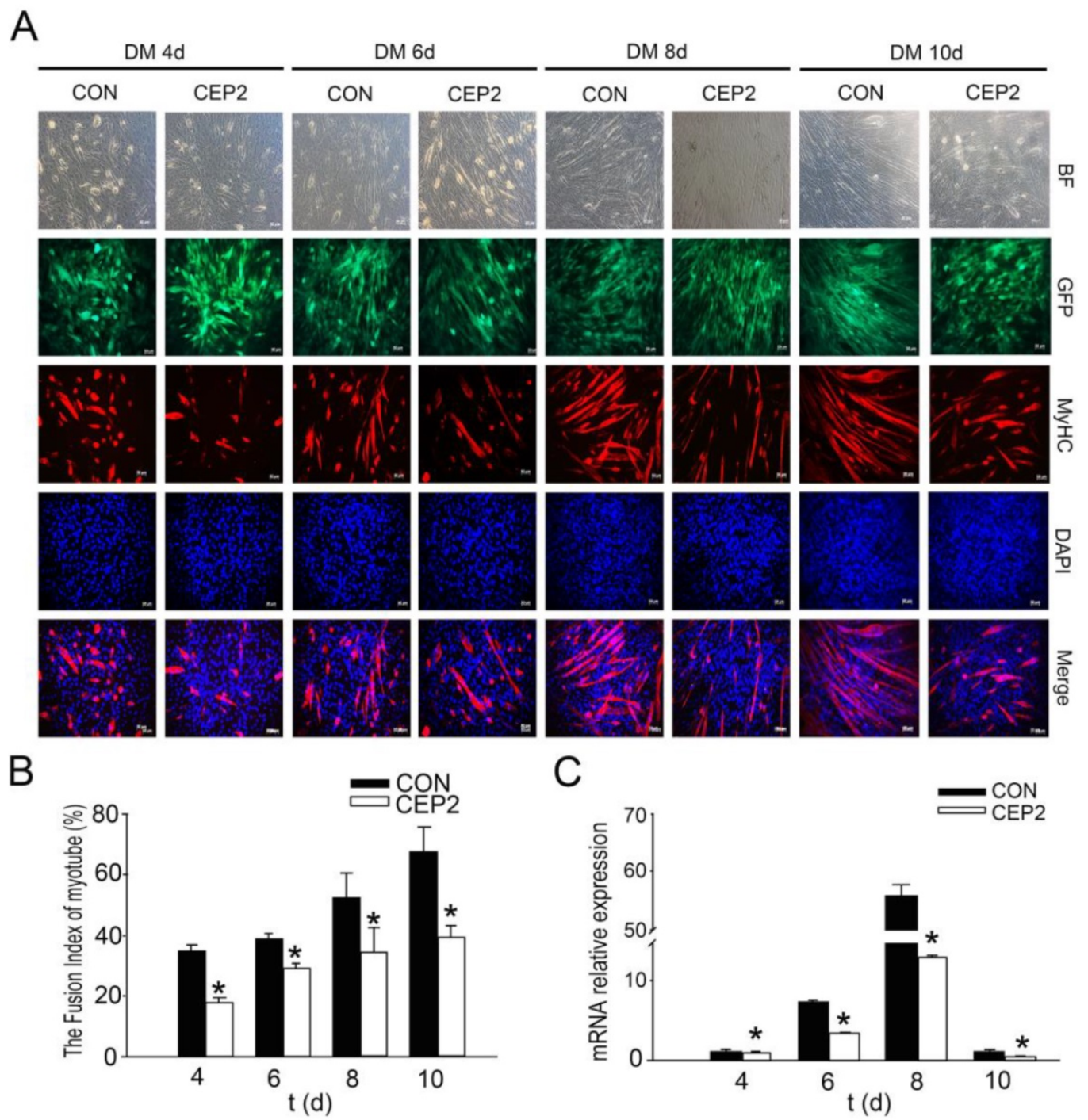

Figure 4. CEP2 inhibited the expression of MyHC during differentiation. (A) MyHC protein was probed by anti-myosin antibody (red) in DM on the $4^{\text {th }}, 6^{\text {th }}$, $8^{\text {th }}$ and $10^{\text {th }} \mathrm{d}^{\text {, }}$ respectively. GFP: represents lentivirus infection efficiency. Scale bar $=50 \mu \mathrm{m}$. (B) The fusion index of myotubes was calculated at four stages. (C) RNA analysis of $M y H C 2 b$ at four stages. $* p<0.05 ; * * p<0.01, \mathrm{n} \geq 3$.

\section{Knockdown of CEP2 enhances myogenesis}

To further confirm the role of CEP2 in attenuating myoblast differentiation, CEP2 knockdown cells were generated using siRNA. As a result, the CEP2 mRNA level was $75 \%$ lower than the control at $24 \mathrm{~h}$ post-transfection (Figure $5 \mathrm{~A}$ ). In this assay, the mRNA level of myf5 and myogenin were increased significantly (Figure 5A), and the protein level Myf5 and MyoD was increased as well (Figure 5B). After differentiation for 4 days, the CEP 2 mRNA level was also significantly lower than the control (Figure 5C). And the RNA level and protein level of myogenic factors showed a significantly increase except for myf5 (Figure $5 \mathrm{C}$ and $\mathrm{D}$ ). The expression of myogenin and MyHC protein were also increased in CEP2 knockdown cells (Figure 5E and F). These results indicated that CEP2 knockdown could enhance myogenesis.

\section{CEP2 cannot influence cell proliferation}

Before cell cycle arrest and fusion into myotubes, the cell cycle of myoblasts undergoes a phase of active proliferation $[5,6]$. The molecular events maintain the balance between proliferation and differentiation of skeletal myoblasts [27], by shedding light on the crosstalk mechanisms taking place between cell cycle regulators and myogenic factors [28-31]. In order to address whether CEP2 gene influences cell proliferation, EdU experiment assay and flow cytometry analysis were performed at $24 \mathrm{~h}$ post-transfection (Figure 6A, B, C and D). It showed that overexpression of CEP2 did not change cell proliferation significantly, which was confirmed by alamlaBlue assay. This assay was performed after transfection with $p c D N A 3.1-C E P 2$ for $6 \mathrm{~h}$ and the dynamic curve of fluorescence values was plotted during cell growth 
(Figure 6E). This finding was similar to the result of experiment in which CEP2 gene was knocked down by siRNA (Supplementary Material: Figure S3). In summary, CEP2 could not influence cell proliferation significantly.

\section{Discussion}

In this study, the effects of CEP2 on myogenesis were investigated. Our results show that the expression of CEP2 is increased during myogenic differentiation. Further, CEP2 overexpression led to attenuating myogenic differentiation, while knockdown of CEP2 resulted in enhancing myogenesis. For the first time, we report here that CEP2 attenuates the myoblast differentiation by suppressing MRFs.

Taken together, these results allow us to propose a model demonstrating the biological role of CEP2 in myogenesis (Figure 7). In our model, when GM was changed to DM, myoblasts express myf5 and myoD which resulted in termination of myoblast proliferation and the expression of myogenin, MRF4, and $M y H C$. Up-regulation of CEP2 inhibits the expression of MRFs and MyHC, ultimately attenuating myoblast differentiation. On the other hand, knockdown of CEP2 promotes the expression of MRFs and $\mathrm{MyHC}$, and thus stimulating myoblast differentiation.
B
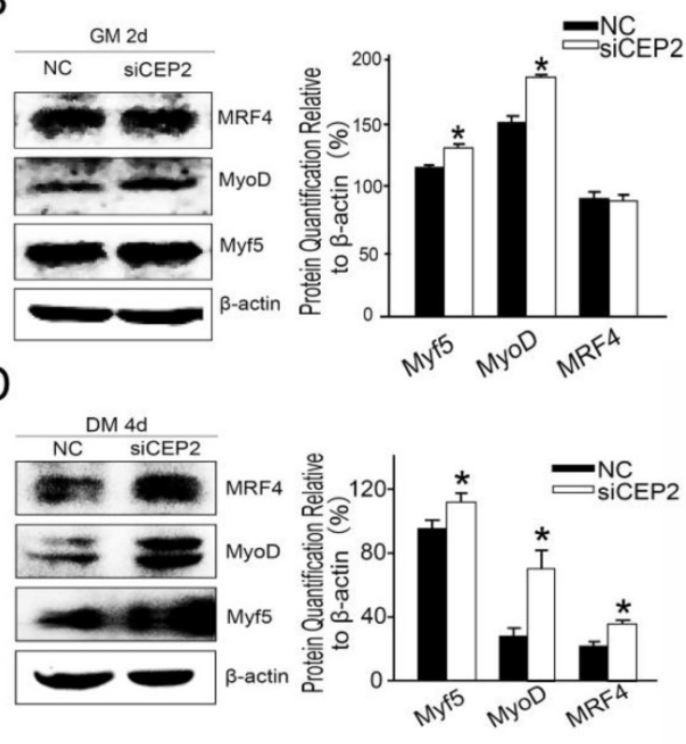

A A
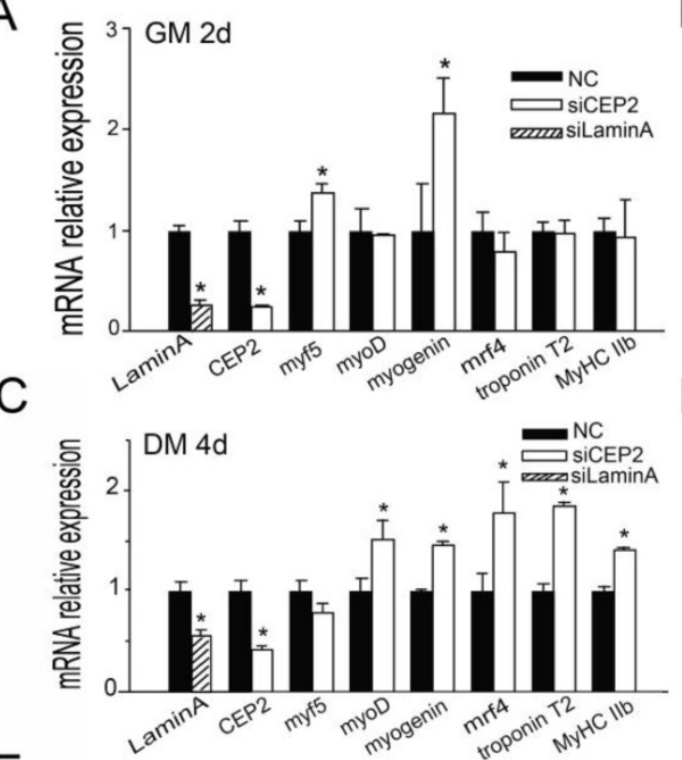

E

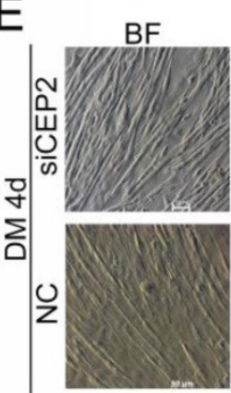

$\mathrm{F}$

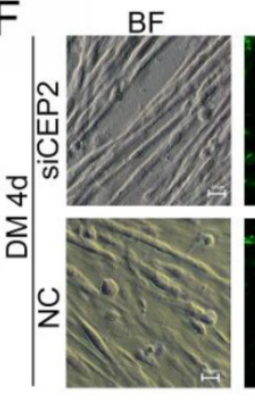

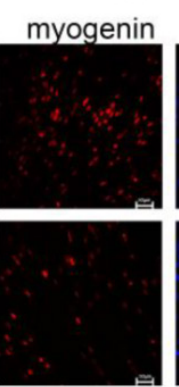

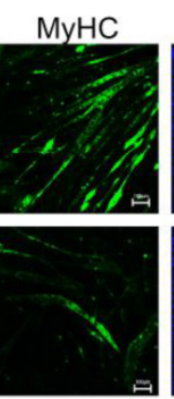

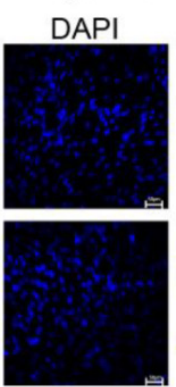

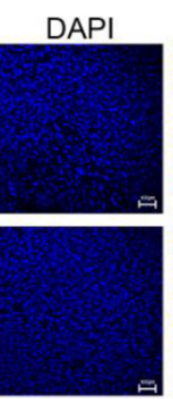

Merge
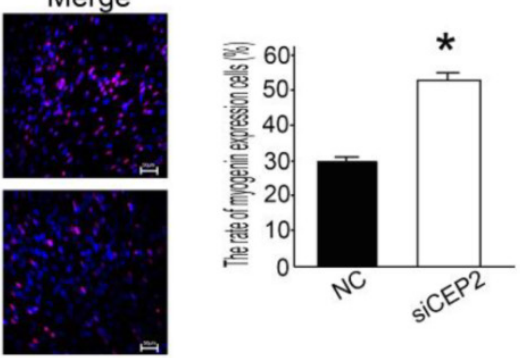

\section{Merge}
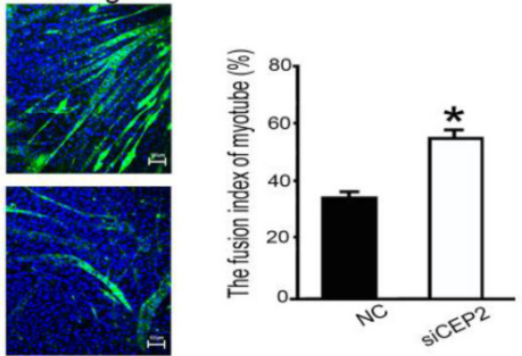

Figure 5. Knockdown of CEP2 enhanced myogenesis. RNA (A) and protein (B) analysis of myogenic factors after transfection of siCEP2 to C2C12 in GM on the $2^{\text {nd }}$ d. siLamin A: positive control (siRNA pool targeted to Lamin A). After differentiation for 4 days, the mRNA (C) and protein (D) of myogenic factors was analyzed. The rate of myogenin expression cells $(E)$ and MyHC (F) were detected with immunofluorescence in DM on the $4^{\text {th }} \mathrm{d}$. Scale bar $=50 \mu \mathrm{m}$. siCEP2: siRNA pool targeted to CEP2, NC: native control (No target siRNA pool). ${ }^{*} p<0.05 ; \mathrm{n} \geq 3$. 
A

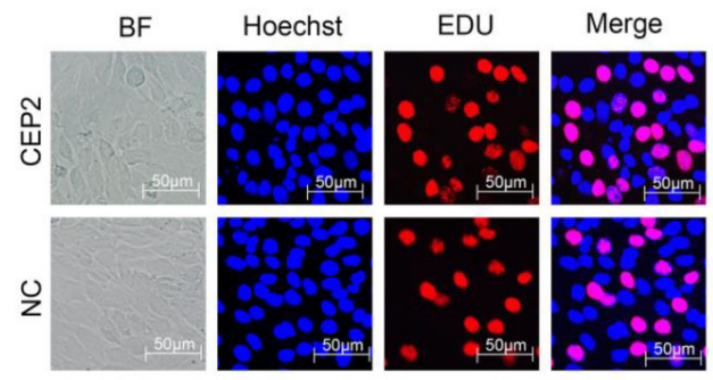

C

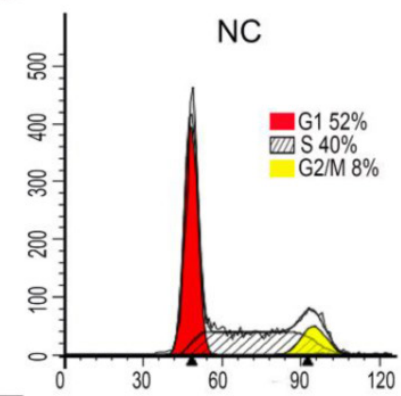

E

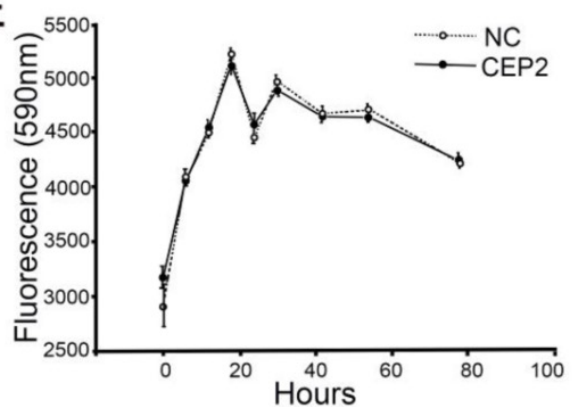

B

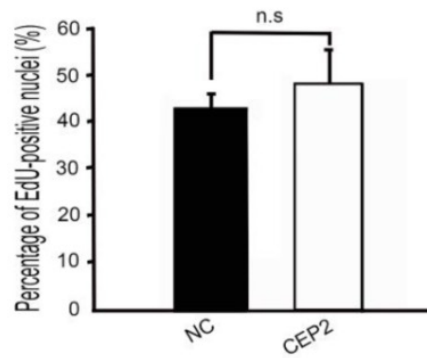

D

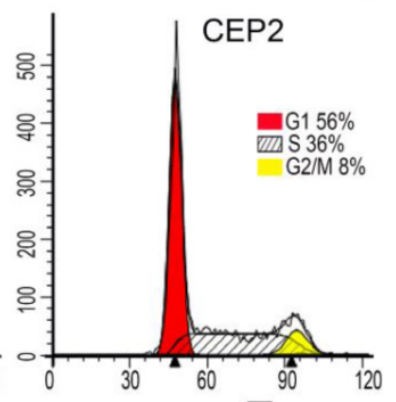

$\mathrm{F}$

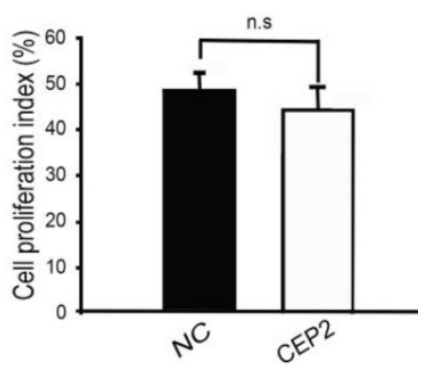

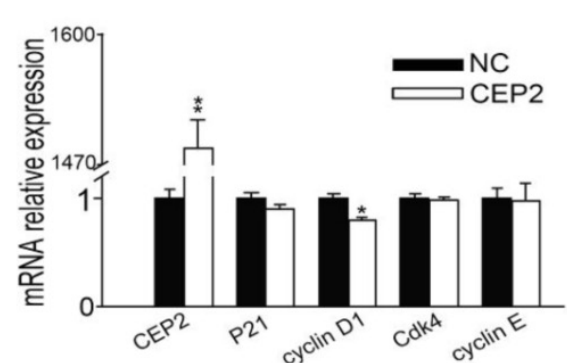

Figure 6. Overexpression of CEP2 did not influence cell proliferation. (A) The cells were fixed for EDU (red) immunostaining at $24 \mathrm{~h}$ post-transfection. Scale bar $=50 \mu \mathrm{m}$. $(\mathrm{B})$ The proportion of proliferation cells in EDU assay was presented as mean \pm S.E.M $(n=3)$. (C) $C 2 C 12$ cells were collected for PI staining and FACSCalibur cell cycle assay. (D) The proliferative index in FACSCalibur assay was presented as mean \pm S.E.M $(n=3)(E)$ The fluorescence value was measured every 6,12 or 24 hours using alamlaBlue assay after 6 hours post-transfection $(n=6)$. (F) RNA analysis of cell cycle-specific genes at 24 h post-transfection. CEP2: pcDNA3. I-CEP2; NC, negative control ( $p c D N A 3.1)$. * $p<0.05 ; \mathrm{n} \geq 3$.

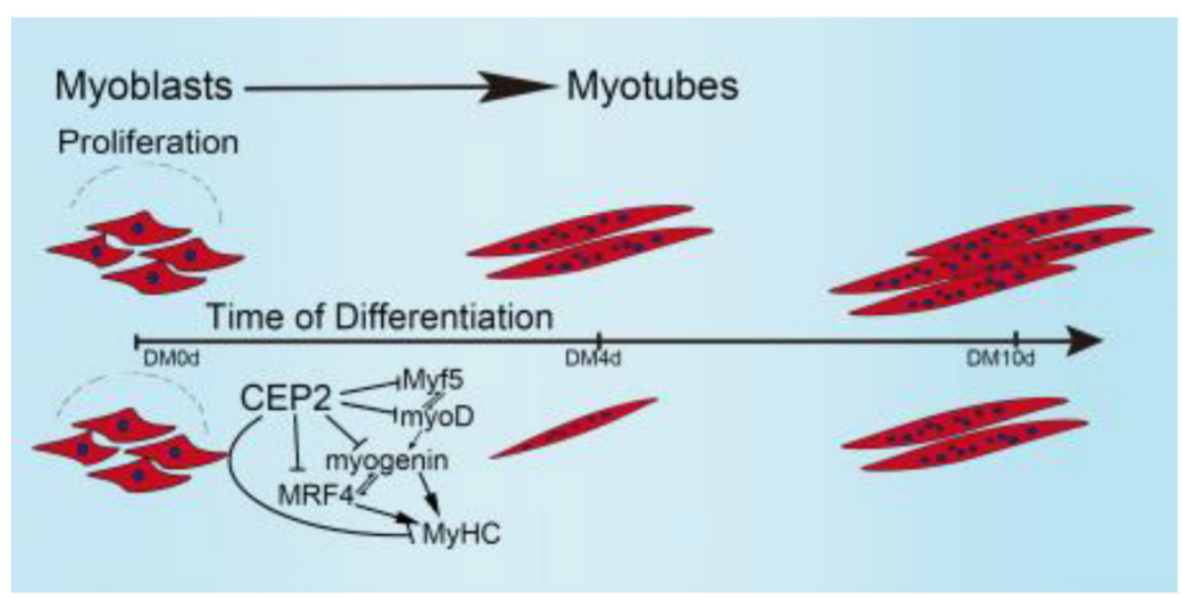

Figure 7. A model illustrating the biological role of CEP2 in myogenesis. DM: differentiation medium.

Critical for skeletal muscle differentiation are the down-regulation of cell cycle activators such as cyclins and cdks [9], and the up-regulation of cell cycle inhibitors such as p21 and p57 [5, 9, 27]. Crosstalk between cell cycle regulators and myogenic factors is important during myoblast differentiation $[5,6]$. Cyclin E-Cdk2 phosphorylates the Ser200 of MyoD, which in turn causes degradation of $\mathrm{MyoD}$ and promotion of late G1-Phase [29]. Cyclin D1 can regulate MyoD expression and the onset of myogenesis by 
controlling the subcellular location of $\mathrm{Cdk} 4$ protein [27]. We investigated whether the role of CEP2 in myogenic differentiation was caused by crosstalk between cell cycle regulators and MRFs. Unexpectedly, neither overexpression nor knockdown of CEP2 significantly influenced cell cycle progression or cell proliferation. Even though cyclin D1 and p21 expression levels had changed, they didn't significantly influence cell cycle events in our investigation. It suggested that CEP2 attenuating myoblast differentiation does not mainly via regulatory mechanisms between cell cycle regulators and myogenic factors.

The effect of CEP2 (a Rho GTPase) on myogenic differentiation is mediated by MRFs in our research. CEP2 overexpression significantly inhibited MRFs expression while CEP2 knockdown markedly up-regulated myogenic factor such as MyoD. This study demonstrated that CEP2 is a repressor of myogenesis. CEP2 is a member of the CEP proteins family, most of which act as Cdc42 effector proteins via a CRBI domain, which binds Cdc42 [20]. Cdc42 is essential for differentiation and myoblast fusion in vivo and vitro [17-19, 32]. Cdc42 inhibits the expression of myogenin, troponin $\mathrm{T}$ and $\mathrm{MyHC}$, which ultimately blocks myogenesis via activation of the C-jun N-terminal kinase (JNK) pathway $[32,33]$. However, it has been suggested that Cdc42 exerts distinct functions under different conditions: For example, Cdc42 has a dual function in myogenesis due to its activation of the stress-activated protein kinase (SAPK) as well as p38, which both are necessary for myogenesis [13, 32].

Pseudopodia formation cannot be induced with a Cdc42/Rac interactive binding domain mutant of CEP2 or a dominant negative Cdc42 with CEP2 in fibroblasts [20]. These results suggest that CEP2 regulates myogenic differentiation through acting as a downstream factor of Cdc42. It showed that CEP2 attenuating myogenesis could be mediated by JNK pathway, which has a crosstalk with MRFs and finally attenuates myogenesis. Additionally, a series of other mechanisms are involved in CEP2-attenuated myogenic differentiation. It has been shown that expression of CEP2 reduces levels of E-cadherin at adherens junctions and alters the organization of F-actin and Vinculin $[12,20]$. This way, cytoskeletal proteins and the extracellular matrix are also involved in myoblast differentiation [34-36].

\section{Supplementary Material}

Figures S1-S3, Tables S1-S2.

http://www.ijbs.com/v11p0099s1.pdf

\section{Acknowledgments}

This research was supported by the Natural
Science Foundation of China (U1201213, 31272417) and the Fundamental Research Funds for the Central Universities (12lgpy44).

\section{Competing Interests}

The authors have declared that no competing interest exists.

\section{References}

1. Rudnicki MA and Jaenisch R. The MyoD family of transcription factors and skeletal myogenesis. Bioessays. 1995; 17: 203-209.

2. Perry RL and Rudnicki MA. Molecular mechanisms regulating myogenic determination and differentiation. Front Biosci. 2000; 5: 750-767.

3. Yokoyama $\mathrm{S}$ and Asahara $\mathrm{H}$. The myogenic transcriptional network. Cell Mol Life Sci. 2011; 68: 1843-1849.

4. Berkes CA and Tapscott SJ. MyoD and the transcriptional control of myogenesis. Semin Cell Dev Biol. 2005; 16: 585-595.

5. Kitzmann M and Fernandez A. Crosstalk between cell cycle regulators and the myogenic factor MyoD in skeletal myoblasts. Cell Mol Life Sci. 2001; 58: 571-579.

6. Lassar AB, Skapek SX and Novitch B. Regulatory mechanisms that coordinate skeletal muscle differentiation and cell cycle withdrawal. Curr opin cell biol. 1994; 6: 788-794.

7. Zabludoff SD, Csete M, Wagner R, et al. p27Kip1 is expressed transiently in developing myotomes and enhances myogenesis. Cell Growth Differ. 1998; 9: $1-11$.

8. Zhang P, Wong C, Liu D, et al. p21 ${ }^{\mathrm{CIP} 1}$ and p57 ${ }^{\mathrm{KIP} 2}$ control muscle differentiation at the myogenin step. Genes Dev. 1999; 13: 213-224.

9. De Falco M and De Luca A. Involvement of cdks and cyclins in muscle differentiation. Eur J Histochem. 2006; 50: 19-23.

10. M.Hindi S, Tajrishi MM and Kumar A. Signaling mechanisms in mammalian myoblast fusion. Sci Signal. 2013; 6: 1-9.

11. Terada K, Misao S, Katase N, et al. Interaction of Wnt signaling with $\mathrm{BMP} /$ Smad signaling during the transition from cell proliferation to myogenic differentiation in mouse myoblast-derived cells. Int J Cell Biol. 2013; 2013: 1-11.

12. Etienne-Manneville $S$ and Hall A. Rho GTPases in cell biology. Nature. 2002; 420: 629-635.

13. Bryan BA, Li D, Wu X, et al. The Rho family of small GTPases: crucial regulators of skeletal myogenesis. Cell Mol Life Sci. 2005; 62: 1547-1555.

14. Doherty JT, Lenhart KC, Cameron MV, et al. Skeletal muscle differentiation and fusion are regulated by the BAR-containing Rho-GTPase-activating protein (Rho-GAP), GRAF1. J Biol Chem. 2011; 286: 25903-25921.

15. Charrasse S, Causeret M, Comunale F, et al. Rho GTPases and cadherin-based cell adhesion in skeletal muscle development. J Muscle Res Cell Moti. 2003; 24: 309-313.

16. Takano H, Komuro I, Oka $\mathrm{T}$, et al. The Rho family $\mathrm{G}$ proteins play a critical role in muscle differentiation. Mol Cell Biol. 1998; 18: 1580-1589.

17. Heller H, Gredinger E and Bengal E. Rac1 inhibits myogenic differentiation by preventing the complete withdrawal of myoblasts from the cell cycle. J Biol Chem. 2001; 276: 37307-316.

18. Abmayr SM and Pavlath GK. Myoblast fusion: lessons from flies and mice. Development. 2012; 139: 641-656.

19. Abramovici $\mathrm{H}$, Gee $\mathrm{SH}$. Morphological changes and spatial regulation of diacylglycerol kinase- $\zeta$, syntrophins, and Rac1 during myoblast fusion. Cell moti cytoskeleton. 2007; 64: 549-567.

20. Hirsch DS, Pirone DM and Burbelo PD. A new family of Cdc42 effector proteins, CEPs, function in fibroblast and epithelial cell shape changes. J Biol Chem. 2001; 276: 875-883.

21. Davie JK, Cho JH, Meadows E, et al. Target gene selectivity of the myogenic basic helix-loop-helix transcription factor myogenin in embryonic muscle. Dev Biol. 2007; 311: 650-664.

22. Han $Y C$, Park $C Y$, Bhagat $G$ et al microRNA-29a induces aberrant self-renewal capacity in hematopoietic progenitors, biased myeloid development, and acute myeloid leukemia. J Exp Med.. 2010; 207(3): 475-489.

23. Zhou L, Wang L, Lu L, et al. A novel target of microRNA-29, Ring1 and YY1-binding protein (Rybp), negatively regulates skeletal myogenesis. J Biol Chem. 2012; 287: 25255-25265

24. Laframboise W, Petrosko P, Krill-Burger J, et al. Proteins secreted by embryonic stem cells activate cardiomyocytes through ligand binding pathways. J Proteomics. 2010; 73(5): 992-1003.

25. Joberty G, Perlungher RR, Macara IG. The Borgs, a new family of Cdc42 and TC10 GTPase-interacting proteins. Mol Cell Biol. 1999; 19(10): 6585-6597.

26. Joberty G, Perlungher RR, Sheffield PJ, et al. Borg proteins control septin organization and are negatively regulated by Cdc42. Nat Cell Biol. 2001;3(10): 861-866.

27. Zhang JM, Qin W, Zhao XH, et al. Coupling of the cell cycle and myogenesis through the cyclin D1-dependent interaction of MyoD with cdk4. EMBO J. 1999; 18: 926-933. 
28. Guo K and Walsh K. Inhibition of myogenesis by multiple Cyclin-Cdk complexes: coordinate regulation of myogenesis and cell cycle activity at the level of E2F. J Biol Chem. 1997; 272: 791-797.

29. Tintignac LA, Leibovitch MP, Kitzmann M, et al. Cyclin E-cdk2 phosphorylation promotes late G1-phase degradation of MyoD in muscle cells. Exp Cell Res. 2000; 259: 300-307.

30. Puri PL, Balsano C, Burgio VL, et al. MyoD prevents cyclinA/cdk2 containing E2F complexes formation in terminally differentiated myocytes. Oncogene. 1997; 14: 1171-1184.

31. Zhang JM, Zhao XH, Qin $\mathrm{W}$, et al. Direct inhibition of G1 cdk kinase activity by MyoD promotes myoblast cell cycle withdrawal and terminal differentiation. EMBO J. 1999; 18: 6983-6993.

32. Meriane M, Roux P, Primig M, et al. Critical activities of Rac1 and Cdc42Hs in skeletal myogenesis: antagonistic effects of JNK and p38 pathways. Mol Biol cell. 2000; 11: 2513-2528.

33. Vasyutina E, Martarelli B, Brakebusch C, et al. The small G-proteins Rac1 and Cdc42 are essential for myoblast fusion in the mouse. Proc Natl Acad Sci U S A. 2009; 106: 8935-8940.

34. Velleman SG, Shin J, Li XH, et al Review: The skeletal muscle extracellular matrix: possible roles in the regulation of muscle development and growth. Can J Anim Sci. 2012; 92: 1-10.

35. Sequeira V, L.A.M Nijenkamp L, A.Regan J, et al. The physiological role of cardiac cytoskeleton and its alterations in heart failure. Biochim Biophys Acta. 2014; 1838: 700-722.

36. Nayak RC, Chang KH, Vaitinadin NS, et al. Rho GTPases control specific cytoskeleton-dependent functions of hematopoietic stem cells. Immunol Rev. 2013; 256: 255-268. 